\title{
Numerical Simulation of a Mechanically Stacked GaAs/Ge Solar Cell
}

\author{
S. Enayat Taghavi Moghaddam \\ Department of Electrical Engineering \\ Mahshahr Branch, Islamic Azad University \\ Mahshahr, Iran
}

\author{
S. Mehrdad Kankanani \\ Department of Electrical Engineering \\ Mahshahr Branch, Islamic Azad University \\ Mahshahr, Iran
}

\begin{abstract}
In this paper, GaAs and Ge solar cells have been studied and simulated separately and the inner characteristics of each have been calculated including the energy band structure, the internal field, carrier density distribution in the equilibrium condition (dark condition) and the voltage-current curve in the sun exposure with the output power of each one. Finally, the output power of these two mechanically stacked cells is achieved. Drift-diffusion model have been used for simulation that solved with numerically method and Gummel algorithm. In this simulation, the final cells exposed to sun light in a standard AM $1.5 \mathrm{G}$ conditions and temperatures are $300^{\circ} \mathrm{K}$. The efficiency of the proposed structure is $9.47 \%$. The analytical results are compared with results of numerical simulations and the accuracy of the method used is shown.
\end{abstract}

Keywords-Gummel Method; Mechanically Stacked Solar Cell; Numerical Simulation; GaAs/Ge; Drift-Diffusion Method

\section{INTRODUCTION}

Photovoltaic (PV) cells convert solar energy to electrical energy and thus they represent an important aspect of clean energy. Since the power of solar cells' energy conversion efficiency using single junction is limited, the multi-junction structure is mostly used. III-V Multi-junction solar cells have achieved the highest efficiencies of any present photovoltaic technology [1-2]. These classes of photovoltaic cells are often grown directly on a single substrate, and connected in series by tunnel diodes. Despite the advantages of this type of solar cells, they still have problems such as the requirements of current matching, lattice matching and use of tunnel junctions. It seems that a mechanical stack structure is a solution for these problems. Therefore, in this article mechanically stacked multijunction solar cells are considered. The sub-junctions in a mechanical stack are fabricated separately on their individual substrates and then are combined into one single mechanically stacked multi-junction device (mostly by using a transparent adhesive) [3-5]. This cell could obtain $38.5 \%$ under x20 radiation [6]. As mentioned in [7], the optimum structure for these cells (by two separated cells) consists of supernatant cell with energy gap $1.4 \mathrm{eV}$ and a cell with energy gap $0.7 \mathrm{eV}$ so the supernatant cell made of GaAs and the underlying cell made of Ge have been selected.

\section{DIFFUSION-DRIFT MODEL AND NUMERICAL SOLUTION}

There are various models which can be used. Considering to the discussed cell's dimensions and the amount of its layers inject, the diffusion-drift model has been selected. This model consists of three main [8]:

$$
\begin{gathered}
\nabla . \varepsilon \nabla V=-\rho \\
\frac{\partial n}{\partial t}=\frac{1}{q} \nabla J_{n}+U_{n} \\
\frac{\partial n}{\partial t}=-\frac{1}{q} \nabla J_{p}+U_{p} \\
\nabla J_{n}=q n \mu_{n} E+q D_{n} \nabla_{n} \\
\nabla J_{p}=q n \mu_{p} E+q D_{p} \nabla_{p}
\end{gathered}
$$

In the above equations, (1), (2), (3) are Poisson's equation, Electron continuity equation, Holes Continuity equation as respectively and relations (4) and (5) as electron flow equation and holes flow equation. In these relations $\varepsilon$ is the throughput coefficient semiconductor, $\rho$ the space charge density, $V$ the electrical potential, $n, p$ are electrons and holes density, $q$ the electron charge, $J_{p}$ and $J_{n}$ the hole and electron density, $\mu_{n}$ and $\mu_{p}$ the electron and hole mobility coefficient, $E$ the electric field and $D_{p}$ and $D_{n}$ carries the diffusion coefficient. In this paper, three variables $(V, n, p)$ are selected as independent variables, the equations are accordingly adjusted and solved by a numerical method, as described below.

\section{A. Gummel method}

One of the common methods for solving the diffusion-drift equations is the Gummel method [9]. In this algorithm, first Poisson's equation is solved by considering the primary conditions and a distribution for carries in piece length is obtained, and then continuity equations are obtained by this distribution, solved and result to a new value for potential distribution in the piece. This process continues until the intended accuracy is reached. 


\section{B. Discretizing the equations}

For discretization diffusion-drift equations have been used from finite difference method [10]. But in discretization the equations related to semi-conductive simulation it should be considered to one point. Discretization steps should not be larger than a certain limit, otherwise the numerical iterative method is to be divergent and the simulation is not valid. Along with, there are three conditions:

- the limitation related to location steps $\left.\left(\Delta r \leq L_{D}\right)=\sqrt{\frac{\varepsilon k_{B T}}{q^{2} N}}\right)$

- the limitation related to time steps $\left(\Delta t \leq t_{d r}\right)=\frac{\varepsilon}{q \mu N}$.

- the limitation in voltage steps applied to the intended piece $\left(\Delta V \leq V_{t}\right)=\frac{k_{B T}}{q}$.

In above relations, $\Delta r, \Delta V$ and $\Delta t$ are discretization steps of local, time, and applied voltage to piece as respectively. Also $K_{B}$ is the Boltzmann constant, $T$ the environment temperature, $N$ the density of carriers mold, $t_{d r}$ the semi-conductive rest time and $V_{t}$ its heat potential. In addition to the expressed issues, it is necessary to say that since the equations are solved numerically with replication methods (Gummel method), these equations should be written in a way to be suitable for iterative algorithms. Hence, in addition to discretization, it should be rewritten appropriately. So we rewrite one by one the relations to diffusion-drift equations for one-dimension mode. In order to rewrite Poisson's equation, first we discretize the equation (1) and replace the relation $\rho=p-n+N_{d}-N_{a}\left(N_{d}\right.$ and $N_{a}$ are donor and acceptor impurity densities as respectively) in it in order to write the equation in terms of independent variables. Then we replace $V_{\text {new }}=V_{\text {old }}+\Delta V$ in obtained equation to give a final form for Poisson's equation (6 and 7). This frame can be used in the iterative algorithm.

$$
\begin{aligned}
& A_{i} V_{i+1}^{\text {New }}+B_{i} V_{i}^{\text {New }}+C_{i} V_{i-1}^{\text {New }}=D_{i} \\
& B_{i}=-\left(\frac{2}{\Delta_{x}^{2}}+\frac{q n_{i}}{\varepsilon V_{t}}\left(e^{\alpha}+e^{-\alpha}\right)\right), \frac{D_{i}}{l}=e^{\alpha}-e \alpha-\frac{N^{\prime}}{n_{i}}+\left(e^{\alpha}+e^{-\alpha}\right) A_{i}=C_{i}=\frac{1}{\Delta x^{2}} \\
& \alpha=\frac{V_{i}^{\text {Old }}}{V_{t}}, l=\frac{q n_{i}}{\alpha}, N^{\prime}=N_{d}-N_{a}
\end{aligned}
$$

where $\Delta_{x}$ is the local step in one-dimension and $i$ is the discrete points number along with this dimension. For solving (9), the LU placement method is used (10). However, it has been stated that using (7) and (8) depends the simulation to the discretization step length [11] and if the voltage difference between two subsequently points is higher than $2 V_{t}$ into the piece, the simulation will diverge. Hence, the GummelScharfetter flow relation ( (9) for electrons) is used [12].

$m=\frac{V_{i}-V_{i-1}}{V_{t}} J_{i-1 / 2}^{n}=-\frac{q D_{i-1 / 2}^{n}}{\Delta x^{2}}[n(i) B(m)-n(i-1) B(-m)]$

The aim of $B(m)$ is that Bernoulli function. By replacing (9) in (2) and its discretization, assuming the steady state $\left(\frac{\partial n}{\partial t}\right)=0$ we obtain:

$$
\begin{aligned}
& A n_{i} n_{i+1}+B n_{i} n_{i}+C n_{i} n_{i-1}=R n_{i} \\
& C_{n t}=\frac{D_{i-1 / 2}^{n} B(-m)}{\Delta x^{2}}, B n_{i}=-\frac{1}{\Delta x^{2}}\left[D_{i+1 / 2}^{i} B(n)+D_{i-1 / 2}^{i} B(m)\right] A n_{i}=\frac{D_{i-1 / 2}^{n} B(-n)}{\Delta x^{2}} \\
& n=\frac{V_{i}-V_{i+1}}{V_{t}}, \quad R n_{i}=U n_{i}-G n_{i}
\end{aligned}
$$

Where $U n_{i}$ and $G n_{i}$ are the recombination rate and the rate of electrons production respectively. Similarity, the same process is used for holes.

\section{PROPOSED SOLAR CELL STRUCTURE}

As mentioned in the introduction part, as it is necessary to be the higher cell energy gap as equal $1 / 4 \mathrm{eV}$ (3), semiconductive GaAs to $\mathrm{Eg}=1.41 \mathrm{eV}$ is the best option. The structure of this cell is provided in Figure 1a. The shown cell in Figure 1a is a one-link $p n$ cell that has used the areas with high injection in order to reduce the ohmic effect of contacts. The top surface of the cell is covered by an anti-reflex covering layer to minimize the reflection losses. The thickness of the desired cell is $14 \mu \mathrm{m}$ that is enough for our aim [3]. The underlying cell should have an energy gap of $0.7 \mathrm{eV}$ and semiconductors as InGaAsm, GaSb, and Ge are the best

\begin{tabular}{|c|c|c|}
\hline$p^{*}$ Ge & $0.92 \mathrm{~mm}$ & $9.8 \times 10^{18}$ \\
\hline$p$ Ge & $4.96 \mu \mathrm{m}$ & $6.0 \times 10^{1 \mathrm{k}}$ \\
\hline n Ge & $6.72 \mu \mathrm{m}$ & $2.7 \times 10^{1 \%}$ \\
\hline$n^{*}$ Ge & $1.4 \mathrm{~mm}$ & $9.0 \times 10^{18}$ \\
\hline
\end{tabular}
options for this purpose with energy gaps of $0.67 \mathrm{eV}, 0.72 \mathrm{eV}$, and $0.75 \mathrm{eV}$ respectively. In this paper, Ge has been used for the underlying cell. The structure of this cell is shown in Figure $1 b$.

(b)

Fig. 1. Cell structure in simulation: (a) upper cell (GaAs) (b) underlying cell (Ge) 


\section{NUMERICAL SIMULATION}

The numerical simulations are performed in MATLAB 2010a [13-14]. It is assumed that each absorbed photon provide an electron-hole pairs. Each of the sub-cells is simulated in two states (in balance state (dark) and in light radiation state). Final results are shown in Figure 2. Comparison of analytic and simulation data is shown in Table I. It should be noted that if we connect sub-cells in mechanical layout as parallel to have a separated charge, maximum final cell power is equal to the sum of powers maximum of each cells [3]. According to the simulation, the output maximum power of the GaAs is equal to
$0.635 \mathrm{~mW} / \mathrm{cm}^{2}$ and of the Ge cell to $0.635 \mathrm{~mW} / \mathrm{cm}^{2}$. Hence the efficiency of changing the GaAs/Ge cell with this mechanical layout is $9.47 \%$.

TABLE I. COMPARISON OF ANALYTIC AND SIMULATION DATA

\begin{tabular}{|c|c|c|}
\hline Variable & Simulation results & Analytic results \\
\hline $\mathrm{I}_{\mathrm{SC}(\mathrm{GaAs})}$ & $7.543 \mathrm{~mA}$ & $7.666 \mathrm{~mA}$ \\
\hline $\mathrm{V}_{\mathrm{OC}(\mathrm{GaAs})}$ & $0.9501 \mathrm{~V}$ & $0.9505 \mathrm{~V}$ \\
\hline $\mathrm{I}_{\mathrm{SC}(\mathrm{Ge})}$ & $9.192 \mathrm{~mA}$ & $9.343 \mathrm{~mA}$ \\
\hline $\mathrm{V}_{\mathrm{OC}(\mathrm{Ge})}$ & $0.1300 \mathrm{~V}$ & 0.1304 \\
\hline
\end{tabular}

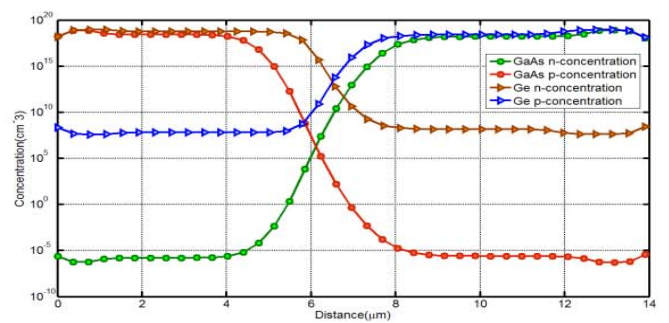

(b)

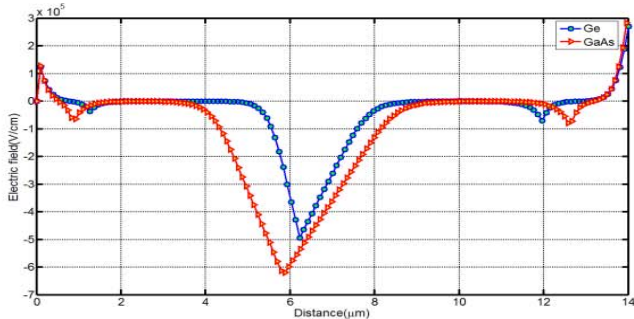

(d)

(c)

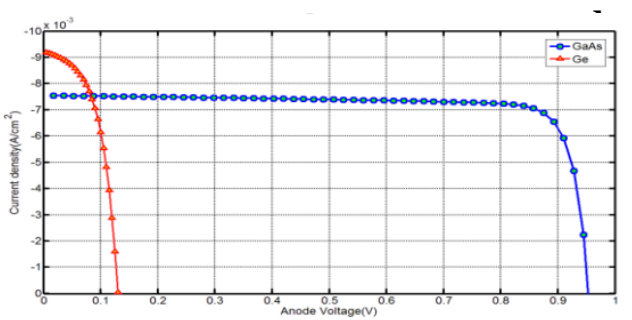

(e)

Fig. 2. Simulation results: )a) conduction band diagram (b) electrical field variations (c) carrier concentration (d) density of electric charge in balance state (dark) (e) I-V characteristics in light radiation state

\section{CONCLUSION}

In this article, a GaAs/Ge solar cell by mechanical layout was simulated in two states, dark and light radiation, with standard conditions AM1.5 G at $300^{\circ} \mathrm{k}$ temperature. For this work we used the diffusion-drift equations that were solved, using numerical methods, in MATLAB. The simulated results are shown and discussed and their values are compared to those from analytical computations.

\section{ACKNOWLEDGMENT}

The author would like to acknowledge the Islamic Azad University, Mahshahr Branch, Iran, for funding the research project entitled "Simulation of hetero-junction GaAs/Ge solar cell” out of which this paper is derived. The authors also acknowledge Mr. Jabar Ganji for his contribution.

\section{REFERENCES}

[1] R. Aguinaldo, Modeling Soluiton and Simulation for Advanced III-V Photovoltaics Based on Nanostructures, M.S. Thesis, Dept. Material 
Science \& Engineering, Rochester Institute of Technology, New York, United States, 2008

[2] F. Dimroth, M. Grave, P. Beutel, U. Fiedeler, C. Karcher, T. N. Tibbits, A. W. Bett, "Wafer bonded four-junction GaInP/GaAs/GaInAsP/GaInAs concentrator solar cells with 44.7\% efficiency”, Prog. Photovolt: Res. Appl., Vol. 22, No. 3, pp. 277-282, 2014

[3] L. D. Partain, M. S. Kuryla, R. E. Weiss, R. A. Ransom, P. S. McLeod, L. M. Fraas, J. A. Cape, " $26.1 \%$ solar cell efficiency for Ge mechanically stacked under GaAs”, Journal of Applied Physics, Vol. 62, No. 7, pp. 3010-3015, 1987

[4] S. Yoshidomi, J. Furukawa, M. Hasumi, T. Sameshima, "Mechanical Stacking Multi Junction Solar Cells Using Transparent Conductive Adhesive”, Advanced Materials and Characterization Techniques for Solar Cells II, Vol. 60, pp. 116-122, 2014

[5] K. Makita, "Highly efficient and reliable mechanically stacked multijunction solar cells using advanced bonding method with conductive nanoparticle alignments”, IEEE 40th Photovoltaic Specialist Conference (PVSC), pp. 0495-0498, 2014

[6] J. D. Mc Cambridge, M. A. Steiner, B. L. Unger, K. A. Emery, E. L. Christensen, M. W. Wanlass, J. W. Ashmead, "Compact spectrum splitting photovoltaic module with high efficiency”, Progress in Photovoltaics: Research and Applications, Vol. 19, No. 3, pp. 352-360, 2011
[7] I. Mathews, W. Yu, D. Gordon, "Mechanically Stacked Solar Cells for Concentrator Photovoltaics”, International Conference on renewable Energy and Power Quality. Spain, Vol. 19, No. 1, pp. 627-628, 2011.

[8] A. Jungel, Transport Equations for Semiconductors, Springer, 2009

[9] H. K. Gummel, “A self-consistent iterative scheme for one-dimensional steady state transistor calculations", IEEE Transactions on Electron Devices, Vol. 11, No. 10, pp. 455-465, 1964

[10] M. N. O. Sadiku, “Monte Carlo Methods for electromagnetics”, CRC Press, Boca Raton, 2009

[11] D. Vasileska, S. M. Goodnick, G. Klimeck, “Computational Electronics: semiclassical and quantum device modeling and simulation”, CRC Press, Boca Raton, 2010

[12] D. L. Scharfetter, D. L. Gummel, "Large signal analysis of a silicon read diode oscillator”, IEEE Transactions on Electron Devices, Vol. 16, No. 1, pp. 64-77, 1969

[13] S. L. Chuang, Physics of Optoelectronic Devices, Wiley, 1995

[14] S. M. Kankanan, "Numerical Simulation of Multijuction GaAs Solar Cell”, MSc. Thesis, Electrical Engineering, Faculty of Engineering, Shahid Chamran Universitu of Ahwaz, 2015 